\title{
Continuous cognitive dynamics of the evaluation of trustworthiness in Williams syndrome
}

\author{
Marilee A. Martens ${ }^{1,2}{ }^{*}$, Adam E. Hasinski ${ }^{3}$, Rebecca R. Andridge ${ }^{4}$ and William A. Cunningham ${ }^{3}$ \\ ${ }^{1}$ Department of Psychology, The Ohio State University-Newark, Newark, OH, USA \\ ${ }^{2}$ Nisonger Center, The Ohio State University, Columbus, OH, USA \\ ${ }^{3}$ Department of Psychology, The Ohio State University, Columbus, OH, USA \\ ${ }^{4}$ Department of Biostatistics, The Ohio State University, Columbus, OH, USA
}

Edited by:

Helen Tager Flusberg, Boston

University, USA

Reviewed by:

Klaus Libertus, Kennedy Krieger Institute, USA

Gary Morgan, City University London, UK

\section{${ }^{*}$ Correspondence:}

Marilee A. Martens, Department of Psychology, The Ohio State University-Newark, 2012 Founders, 1179 University Dr. Newark, $\mathrm{OH}$ 43055, USA.

e-mail:martens.22@osu.edu
The decision to approach or avoid an unfamiliar person is based in part on one's evaluation of facial expressions. Individuals with Williams syndrome (WS) are characterized in part by an excessive desire to approach people, but they display deficits in identifying facial emotional expressions. Likert-scale ratings are generally used to examine approachability ratings in WS, but these measures only capture an individual's final approach/avoid decision. The present study expands on previous research by utilizing mouse-tracking methodology to visually display the nature of approachability decisions via the motor movement of a computer mouse. We recorded mouse movement trajectories while participants chose to approach or avoid computer-generated faces that varied in terms of trustworthiness. We recruited 30 individuals with WS and 30 chronological age-matched controls (mean age $=20$ years). Each participant performed 80 trials (20 trials each of four face types: mildly and extremely trustworthy; mildly and extremely untrustworthy). We found that individuals with WS were significantly more likely than controls to choose to approach untrustworthy faces. In addition, WS participants considered approaching untrustworthy faces significantly more than controls, as evidenced by their larger maximum deviation, before eventually choosing to avoid the face. Both the WS and control participants were able to discriminate between mild and extreme degrees of trustworthiness and were more likely to make correct approachability decisions as they grew older. These findings increase our understanding of the cognitive processing that underlies approachability decisions in individuals with WS.

Keywords: Williams syndrome, hypersociability, mouse-tracking

\section{INTRODUCTION}

Evaluations of facial expressions are critical for assessing the approachability of unfamiliar individuals (Winston et al., 2002). Individuals who have Williams syndrome (WS) appear to indiscriminately approach unfamiliar individuals, a feature characterized as hypersociability, and also exhibit deficits in the processing of faces. This combination makes them a critical population for both basic science, where their deficits can inform general theories of person perception and approach-avoid processes, as well as clinical research, as their deficits can leave them vulnerable in some social contexts. The present research seeks to examine the dynamic nature of approachability judgments from facial cues in both individuals with WS and controls.

Individuals with WS display a distinctive and atypical cognitive, behavioral, and neuroanatomical profile. WS has been described as an ideal model in which to investigate the neural substrates of human cognition and behavior. WS is a genetic neurodevelopmental disorder with an estimated prevalence rate of 1 in 7,500 (Stromme et al., 2002), caused by a hemizygous deletion of approximately 26 genes on the long arm of chromosome 7 (Peoples et al., 2000). Individuals with WS typically display a mild to moderate intellectual delay. Relative strengths have been noted in particular aspects of language such as receptive vocabulary and fluency (Don et al., 1999; Mervis and Klein-Tasman, 2000; Robinson et al., 2003; Clahsen et al., 2004; Vicari et al., 2004; see Brock, 2007 for a review), however cross-linguistic studies have shown that the grammatical abilities of individuals with WS are at or below the level of CA- and MA-matched children with intellectual disabilities (other than Down syndrome) as well as MA-matched typically developing (TD) children (Gosch et al., 1994; Volterra et al., 1996; Lukács et al., 2001; Volterra et al., 2003). Individuals with WS have specific deficits in visuospatial skills (Porter and Coltheart, 2006), as well as delays in motor development. Abnormal muscle tone has been documented in individuals with WS, as evidenced by hypotonia in WS children and hypertonia in WS adults (Chapman et al., 1996). Delays in gross and fine motor coordination have also been noted in individuals with WS (Atkinson et al., 1997; Elliott et al., 2006; Hocking et al., 2011). A salient behavioral feature displayed by individuals with WS is their hypersociability, characterized by an excessive desire to meet people, and a lack of stranger anxiety (Bellugi et al., 1999; Frigerio et al., 2006). 
The increased sociability in WS has been associated in part with poor emotion identification of facial expressions (Jarvinen-Pasley et al., 2010). TD children as young as 5 years are able to use facial features to make judgments of affective expression and it is these facial features which continue to be used to make rapid judgments of trustworthiness (Cunningham and Odom, 1986; Cowell and Stanney, 2002; Want et al., 2003; Willis and Todorov, 2006). Children with WS show delays in processing happy and sad emotions (Karmiloff-Smith et al., 1995), as well as angry and scared emotions (Porter et al., 2007). Individuals with WS also show deficits matching facial expressions (Levy et al., 2011), focusing attention on angry faces (Santos et al., 2010), and recognizing angry faces (Porter et al., 2010). However, the effect that this perceptual deficit has on social evaluations is not well understood.

The hypersociability displayed by individuals with WS has typically been examined using approachability tasks in which photographs of unfamiliar faces are individually rated on a Likert-scale to determine how approachable they appear. These studies have produced conflicting findings depending on the nature of the task stimuli and whether or not the facial stimuli displayed specific emotions. Individuals with WS rated "positive" (trustworthy and approachable) and "negative" (untrustworthy and unapproachable) faces as more approachable than chronological age-matched controls (Jones et al., 2000; Martens et al., 2009). When viewing faces depicting specific positive and negative emotions, such as happiness, anger, or fear, individuals with WS rated only the happy faces as more approachable (Frigerio et al., 2006). While these behavioral measures help increase our understanding of hypersociability in WS, they do not provide an opportunity to explore the dynamics of cognitive processing as approachability decisions are made.

Cognition is a continuous, dynamic process and static measures which only examine a participant's final choice do not capture this fluidity (Spivey and Dale, 2006). Converging evidence from continuous measures such as eye-tracking suggest that compared to controls, individuals with WS show increased gaze durations when looking at strangers (Mervis et al., 2003), when viewing facial expressions (Porter et al., 2010), and during cognitively challenging tasks (Doherty-Sneddon et al., 2009). Additional research has shown that individuals with WS take longer than TD individuals to disengage from looking at faces than objects (Riby et al., 2011). While these studies highlight abnormalities in perceptual processing associated with WS, they do not help explain WS-related differences in evaluating face stimuli or their hypersociability more generally.

In the current study, we chose to explore hypersociability in WS by examining the dynamic cognitive processing that occurs when individuals are asked to approach or avoid faces of unfamiliar persons via mouse responses. A burgeoning literature suggests that the continuous nature of cognitive processes can be visually displayed via mouse-tracking - the motor movement of a computer mouse as a decision is being made - (Gold and Shadlen, 2001; Shin and Rosenbaum, 2002; Dale et al., 2007). Each decision trial provides continuous data which graphically displays underlying aspects of cognition (Magnuson, 2005). Arm movements that are made when controlling a computer mouse can be adjusted in the process of making a choice and mouse-tracking allows one to visually observe the effects of an alternate choice that may be competing with the correct response. For example, it has been demonstrated that when individuals are shown two objects in opposite corners of a computer screen and are asked to click on the object that is named, the participants will show more attraction (mouse movement) toward the competing object if the object name and the competing name start with the same sound (candle and candy) than with different sounds (candle and jacket) (Spivey et al., 2005). The technique has also been used to investigate gender stereotypes (Freeman and Ambady, 2009), perceptions of race (Wojnowicz et al., 2009; Freeman et al., 2010), and the social categorization of sex (Freeman et al., 2008). This methodology is fairly recent and as such is just beginning to be utilized with individuals who have intellectual disabilities. Its use in this particular study allows us to make more advanced inferences about aspects of cognitive processing that occur as approachability decisions are made in individuals with WS.

\section{MATERIALS AND METHODS PARTICIPANTS}

Thirty individuals with WS (mean age $=20.8$ years, range $=8-$ 41 years) and 30 chronological age-matched TD controls (mean age $=20.9$ years, range $=8-42$ ) participated in the study. The majority of the data collection from the participants with WS occurred during a WS Syndrome National Convention, with the remaining WS participants recruited locally. The control participants were recruited from the community and from siblings of the WS participants. All of the WS and control participants were Caucasian and right-handed. Appropriate institutional IRB approval was obtained and consent was given by either the participants or their guardians. The parents/guardians all affirmed that their child was comfortable using a computer mouse. Their familiarity with the computer was confirmed by the experimenter, who observed that all participants demonstrated immediate ease at manipulating the computer mouse. The participant demographics are displayed in Table 1.

\section{STIMULI}

Our stimuli came from a previously published computergenerated face set (Oosterhof and Todorov, 2008), derived using FaceGen Modeller (Inversions, 2007). This face set consists of a series of bald, Caucasian, male faces of European ethnicity. Each face is depicted multiple times with systematic variations that change the perceived approachability/trustworthiness of the face, with inferences of trustworthiness based on similarity to expressions signaling approach or avoidance behavior (Todorov et al.,

\section{Table 1 | Participant demographics.}

\begin{tabular}{lllll}
\hline Characteristic & Overall & WS & Control & $P$-value \\
\hline$N$ & 60 & 30 & 30 & \\
Age (years) & $20.8(10.0)$ & $20.8(10.1)$ & $20.9(10.0)$ & 1.00 \\
IQ & $91.3(22.2)$ & $73.9(14.2)$ & $108.8(13.1)$ & $<0.0001$ \\
Female gender, N(\%) & $38(63 \%)$ & $21(70 \%)$ & $17(57 \%)$ & 0.28 \\
\hline
\end{tabular}

Results are presented as mean (SD) except where noted. 
2008). We selected 20 faces from the set and used four variations of each, based on their standard deviation (SD) from the average rating: extremely untrustworthy $(-4.5 \mathrm{SD})$; mildly untrustworthy $(-1.5 \mathrm{SD})$; mildly trustworthy $(+1.5 \mathrm{SD})$; and extremely trustworthy $(+4.5 \mathrm{SD}$; see Figure 1$)$. These variations resulted in a total of 80 facial stimuli, creating a 2-by-2 design (trustworthiness by extremity). These stimuli were chosen because they lack many irrelevant features of more naturalistic face images (e.g., hair or accessories) that can distract individuals with WS and interfere with their attention to facial expressions (Martens et al., 2009; Capitão et al., 2011).

\section{APPARATUS}

The stimuli were shown on a Dell 3700 Vostro laptop computer, with a screen size of $44 \mathrm{~cm}$. The participants sat at a desk approximately $46 \mathrm{~cm}$ from the computer screen. The target responses (Green or Red circle) were $6.4 \mathrm{~cm}$ in diameter and the face images were $9.6 \mathrm{~cm}$ tall and $5.7 \mathrm{~cm}$ wide. To get a measure of participants' decision processes across time regarding the trustworthiness of the faces, we tracked their mouse cursor movements as they selected response options in regard to whether they would like to approach or avoid each face. We used the MouseTracker software package (Freeman and Ambady, 2010); available at http://mousetracker.jbfreeman.net, which is a self-contained program which presents and records participants' responses.

\section{PROCEDURE}

Participants were presented stimuli in four blocks of 20, with stimuli randomized within each block. ${ }^{1}$ On each trial, participants were presented with a single face. Participants were instructed to indicate whether they wished to go up and talk to the individual, by moving the cursor from the bottom center of the screen to select the corresponding circle on the screen (a green circle to approach and a red circle to avoid). A colored green or red circle was placed in the upper left and right corners of the screen, counter balanced across participants (see Figure 2). Prior to beginning the first block, participants acquired familiarity with the decision task via a practice block using unrelated stimuli (food).

${ }^{1}$ This design allowed participants who became fatigued to take a break or withdraw from the study without tainting their previously collected data. All four blocks were completed by all control participants and by all but one WS participant.
A face appeared only after participants clicked the mouse on the word "start" to begin each trial. The inter-stimulus interval was $1 \mathrm{~s}$ for the red and green circles to reappear and $2 \mathrm{~s}$ for the word "start" to appear. Participants were instructed to respond as fast as possible to each stimulus. If participants did not begin moving the mouse within $750 \mathrm{~ms}$ of the stimulus onset, or if they did not make a response within $8000 \mathrm{~ms}$, they were encouraged to begin moving more quickly and that trial was not logged. The majority of trials were valid, with $99.5 \%$ of control participants' trials and $97.2 \%$ of WS participants' trials producing valid responses.

For analysis, some conditions were mirrored such that all trajectories (including incorrect responses) were made to the upper right corner. Consistent with MouseTracker conventions, trajectories were normalized spatially, to a 4 -by- 3 aspect ratio, and temporally, into 101 time steps. The MouseTracker package computes a summary measure for each trajectory called maximum deviation (MD). MD is a common metric for gauging competition between responses. MD quantifies how far a trajectory deviates toward one option before the participant ultimately settles on the alternative. Thus larger MDs are presumed to indicate greater response competition and more difficulty in making a decision. Following this

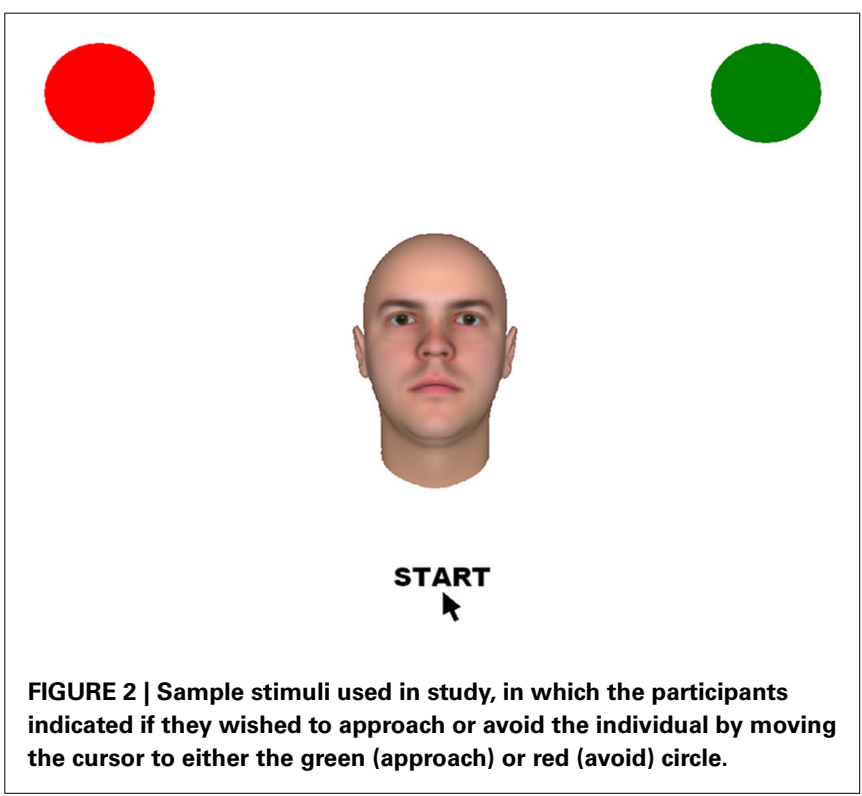

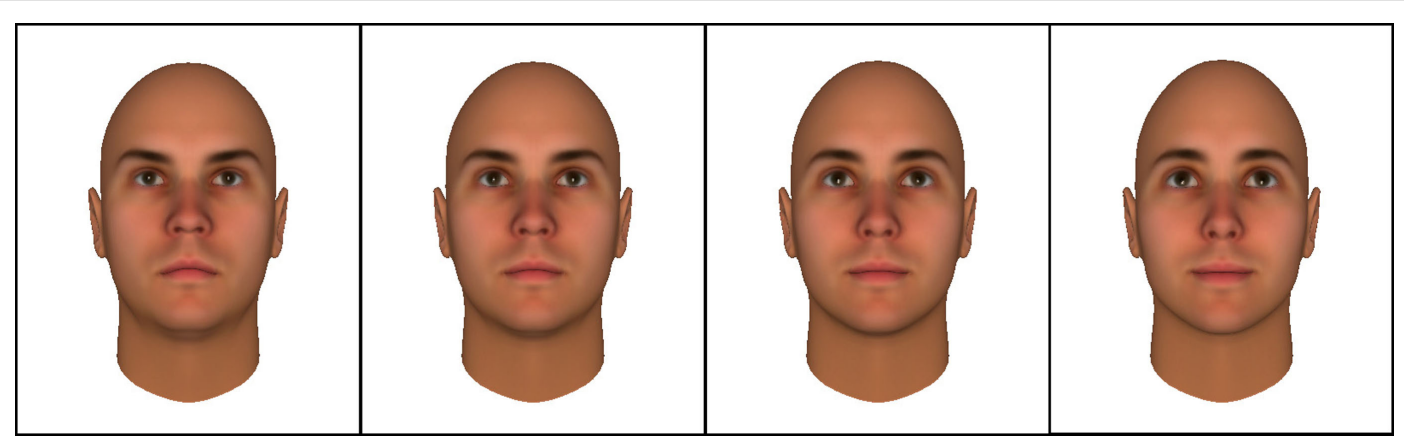

FIGURE 1 | Example of facial stimuli, ranging from "Very untrustworthy" to Very trustworthy (left to right). 
task, the individuals were given the Kaufman Brief Intelligence Test, 2nd edition (Kaufman and Kaufman, 2004) in order to gain a measure of their overall ability level.

\section{DATA ANALYSIS}

The expected behavior was for participants to choose to approach trustworthy faces and avoid untrustworthy faces. However, the participants did not always respond as expected, and therefore, trials were categorized as "correct" or "incorrect." An analysis of incorrect trials was important, given that the hypersociability aspect of the WS phenotype suggests that there may be differences in the ways that errors are generated. Therefore, analysis investigated factors influencing the frequency with which participants responded in a typical fashion, as well as the MD of both correct and incorrect trials.

Linear and generalized linear mixed models were used to test differences between the WS participants and control participants in the rates of correct responses and the MD (Diggle et al., 2002). This type of analysis allows use of individual trial data, taking into account the fact that trials by the same participant were correlated. A compound-symmetric variance-covariance structure for within-participant correlation was estimated by including a random participant-specific intercept in each model. A logistic mixed model was used to model the frequency of correct responses, using the PROC GLIMMIX procedure in SAS 9.3 with a RANDOM statement, and a linear mixed model was used to model the MDs using the PROC MIXED procedure with a RANDOM statement (SAS Institute, Cary, NC, USA). Since not all trials were completed by all participants, the Kenward-Roger approximation to the degrees of freedom was used to bring Type I error rates to nominal levels (Kenward and Roger, 1997), sometimes resulting in non-integer degrees of freedom.

To test for group differences, all models included the effects of group, face type (four level), and their interaction. Models for MD additionally included an indicator for whether the trial resulted in a correct response, and the interaction of this indicator with group, face type, and their interaction. We determined that the MD of the WS participants was toward the alternate choice $84 \%$ of the time (versus toward the outside edge of the computer screen), suggesting that the MD results were not an artifact resulting from errant motor control. To control for potential confounders, adjusted models included the main effects of age, IQ, and reaction time (RT) in milliseconds (ms). Potential effect modification by age was investigated by including the interaction of age and group; this term was not significant in any model and hence was omitted. Gender was not a significant predictor in any models; including it did not impact results and hence was not included in analyses. Orthogonal contrasts were used within the mixed models to estimate adjusted group differences in correct response rates and MD.

\section{RESULTS REACTION TIMES}

The average RT of the WS participants (mean $=1992 \mathrm{~ms}$, $\mathrm{SD}=373 \mathrm{~ms}$ ) was significantly slower than the average RT of the control participants $($ mean $=1759 \mathrm{~ms}, \mathrm{SD}=274 \mathrm{~ms} ; p=0.008)$.

\section{RATES OF CORRECT RESPONSES}

An examination of the correct response rates indicates that both the WS and control participants chose to avoid untrustworthy faces and approach trustworthy faces the majority of the time, but the WS participants did so less often than the control participants. Across all trials, the individuals with WS chose the correct response on $69 \%$ of trials and the control participants chose the correct response on $93 \%$ of trials; both rates were significantly above chance $(p<0.0001)$. Both face type and participant type (WS, control) were significant predictors of correct response ( $p<0.0001$ for both). After adjusting for age, IQ, and RT, there were significant differences in the rates of correctly classified faces between WS and control participants for the extreme faces (Table 2). The odds of a WS participant correctly choosing to avoid an extremely untrustworthy face were 0.39 times the odds of a control participant correctly choosing to avoid an extremely untrustworthy face (95\% CI: 0.23 $0.66, p=0.0004)$. Similarly, the odds of choosing to approach extremely trustworthy faces was significantly lower for WS participants than for controls ( $\mathrm{OR}=0.43,95 \% \mathrm{CI}: 0.27-0.69, p=0.006)$. There was a smaller but borderline significant difference for mildly trustworthy faces, with WS participants less likely than controls to approach mildly trustworthy faces (OR $=0.64,95 \%$ CI: $0.42-0.98, p=0.04)$. There were no significant differences between WS and control participants for mildly untrustworthy faces $(p=0.79)$.

Both WS and control participants had higher rates of correct responses for extreme faces as compared to mild faces. For WS participants, the odds of correct classification of an extreme face was 2.2 times the odds for mild faces (95\% CI: $1.8-2.6, p<0.0001)$, with no difference between trustworthy and untrustworthy faces $(p=0.74)$. For control participants,

Table 2 | Correct response rates and adjusted odds ratios comparing WS participants to control participants.

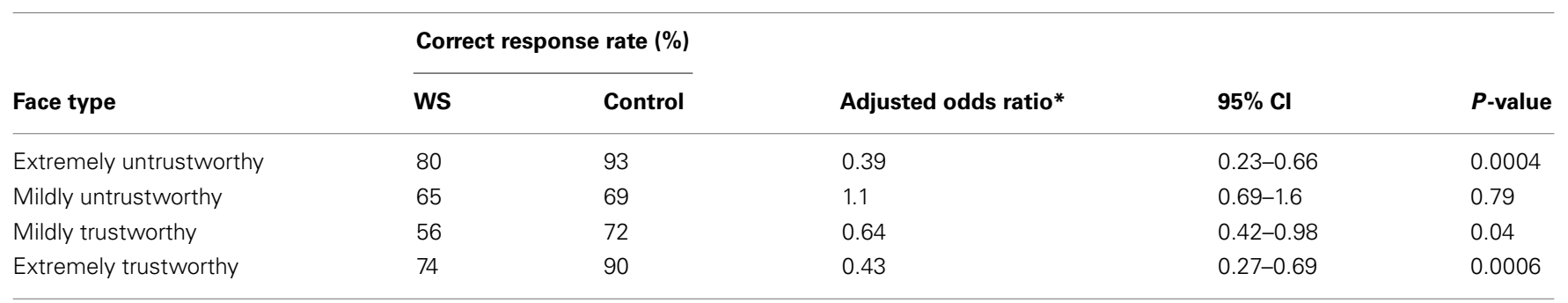

${ }^{*}$ Adjusted for age, 10 , and reaction time. 
the difference in correct response rates between extreme and mild faces depended on whether the face was trustworthy or untrustworthy. For untrustworthy faces, the odds of a control participant correctly classifying an extreme face was 5.7 times the odds of correctly classifying a mild face (95\% CI: 4.0-8.2, $p<0.0001)$, and for trustworthy faces the odds ratio was 3.4 (95\% CI: $2.4-4.6, p<0.0001)$. These two odds ratios were significantly different $(p=0.03)$, showing that it was easier for control participants to differentiate mild versus extreme expressions among the untrustworthy faces, which was not the case for WS participants.

Both age and RT were significantly associated with correct responses. Older participants were more likely to make correct choices, with a 5 year increase associated with $7 \%$ higher odds of selecting the expected response $(p=0.03)$. Shorter RTs were associated with a higher rate of correct responses $(p<0.0001)$.
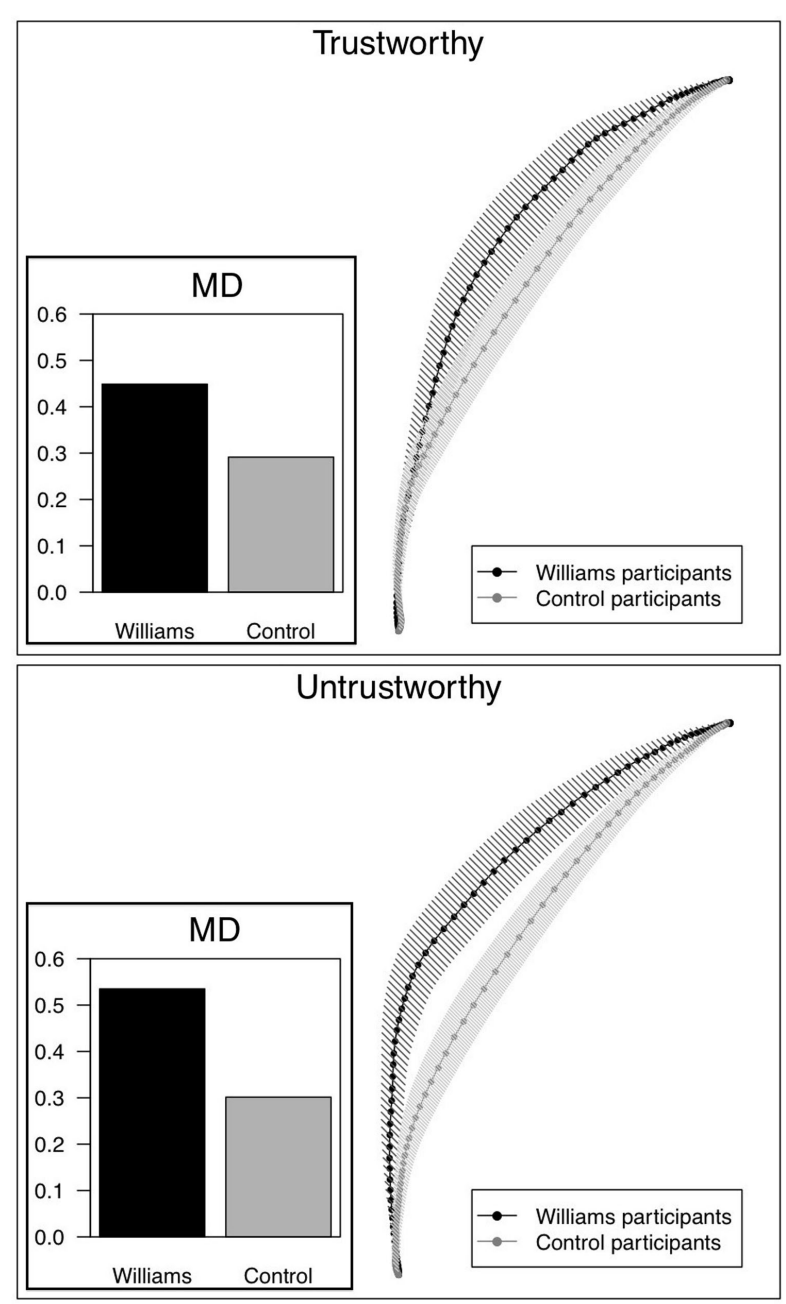

FIGURE 3 | Mean trajectories by face type (trustworthy, untrustworthy) for Williams and control participants, aggregated across extremity of faces and across trials. Shaded region is plus/minus one standard error for each average mouse position. Bar graph shows the adjusted mean maximum deviation for each group of participants.
IQ was not significantly associated with the chance of a correct response.

\section{MAXIMUM DEVIATION FOR CORRECT TRIALS}

For faces where participants did not make an error (75\% of trials overall), WS participants had larger MD on average than control participants, across all face types. Table 3 shows the average MD by participant group and trustworthiness, adjusting for age, IQ, and RT. There was not a significant effect of extremity of faces. Differences in MD between WS and control participants were more pronounced for untrustworthy faces (see Figure 3). WS participants considered approaching untrustworthy faces significantly more than controls, as shown by a significantly larger MD for these faces $(0.53$ vs. $0.30, p=0.005)$. WS participants also considered avoiding trustworthy faces more than control participants, though the difference in MD was of borderline significance ( 0.45 vs. 0.29 , $p=0.06$ ). For WS participants, the tendency to consider approaching the untrustworthy faces was larger than the tendency to avoid the trustworthy face, as evidenced by a significant difference in MD ( 0.53 vs. $0.45, p=0.0001)$; this pattern was not seen in control participants (MD 0.30 vs. $0.29, p=0.61$ ). In other words, individuals with WS were more likely than controls to initially deviate toward untrustworthy faces. In WS participants, the bias to approach untrustworthy faces was larger than the bias to avoid trustworthy faces. No such differences were observed in control participants.

\section{MAXIMUM DEVIATION FOR INCORRECT TRIALS}

For faces where participants did make errors (25\% of trials overall), WS participants considered approaching trustworthy faces that they ultimately chose to avoid significantly more than controls, as shown by a larger MD ( 0.50 vs. $0.27, p=0.01)$, adjusting for age, IQ, and RT (see Table 3). However, there was no significant difference between WS and control participants in their tendency to avoid untrustworthy faces that they ultimately chose to approach (MD 0.38 vs. $0.32, p=0.50$ ). For WS participants, the tendency toward approaching a trustworthy face that was ultimately avoided was larger than the tendency to avoid the untrustworthy face that was ultimately approached, as evidenced by a significant difference in $\mathrm{MD}(0.50$ vs. $0.38, p=0.001)$; this pattern was not seen in control participants (MD 0.27 vs. $0.32, p=0.61$ ). That is to say, WS individuals were more likely than controls to consider approaching trustworthy faces. There was no difference between WS and control participants in the deviation toward avoiding untrustworthy faces. As seen in the correct trials, the approach bias was larger than the avoid bias for WS participants but not for controls.

\section{DISCUSSION}

Individuals with WS display an increased tendency to approach strangers, which makes them vulnerable to exploitation. Hypersociability in WS has typically been examined using behavioral methodologies that incorporate stimuli with varied facial emotional expressions. However, distinguishing facial emotional expression is just one facet of social judgment decision-making (Winston et al., 2002). An individual also needs to decide how trustworthy a person appears before deciding if he/she wants to approach or avoid them. The present study aims to investigate the 
Table 3 | Maximum deviation for WS and control participants, for correct and incorrect trials. Adjusted for age, IQ, and reaction time.

\begin{tabular}{|c|c|c|c|c|c|c|c|}
\hline Trial type & Face type & Participant response & Deviation toward & $\begin{array}{l}\text { Williams } \\
\text { Mean (SE) }\end{array}$ & $\begin{array}{l}\text { Control } \\
\text { Mean (SE) }\end{array}$ & $\begin{array}{l}\text { Difference } \\
\text { Mean (SE) }\end{array}$ & $P$-value \\
\hline \multirow[t]{2}{*}{ Correct } & Untrustworthy & Avoid & Approach & $0.53(0.05)$ & $0.30(0.05)$ & $0.23(0.08)$ & 0.005 \\
\hline & Trustworthy & Approach & Avoid & $0.45(0.05)$ & $0.29(0.05)$ & $0.16(0.08)$ & 0.06 \\
\hline \multirow[t]{2}{*}{ Incorrect } & Untrustworthy & Approach & Avoid & $0.38(0.05)$ & $0.32(0.06)$ & $0.06(0.09)$ & 0.50 \\
\hline & Trustworthy & Avoid & Approach & $0.50(0.05)$ & $0.27(0.06)$ & $0.23(0.09)$ & 0.01 \\
\hline
\end{tabular}

dynamic cognition processing that occurs when individuals with WS are making approach/avoid decisions in response to faces of unfamiliar people who vary in degree of trustworthiness.

An examination of the error rates demonstrated that the individuals with WS were more likely than controls to choose to approach the untrustworthy faces. Furthermore, the real-time motor trajectories revealed that the WS participants considered approaching untrustworthy faces significantly more than controls, as evidenced by their larger MD, before eventually choosing to avoid the face. The control participants appeared to be more definitive in their choice to avoid and did not appear to deviate toward approaching the untrustworthy face as much as the WS participants. These results support evidence indicating that individuals with WS show an approach bias to some negative facial expressions (Bellugi et al., 1999; Jones et al., 2000; Martens et al., 2009; Fishman et al., 2011).

The current findings also support increasing evidence from structural and functional neuroimaging studies that have examined the neural basis of hypersociability in WS. Most imaging studies have focused on the amygdala, which has been shown to be a principal structure in the evaluation of fear (Adolphs, 1995, 2003; Phelps, 2006) and in making social judgments of approachability and trustworthiness (Adolphs et al., 1998; Winston et al., 2002). Increased amygdala volume in individuals with WS has been associated with increased approachability ratings for unapproachable faces (Martens et al., 2009), while functional imaging studies have demonstrated that the amygdala in WS shows decreased reactivity to negative facial expressions (Meyer-Lindenberg et al., 2005; Haas et al., 2009; Plesa-Skwerer et al., 2009).

We also found individuals with WS were more likely than controls to choose to avoid an extremely trustworthy face, and to a lesser degree, a mildly trustworthy face. These results may be associated with the fact that the facial expression on the trustworthy faces showed no teeth and no definitive smile, and therefore may have appeared more neutral than happy (see Figure 1D). Previous results have shown that individuals with WS rate neutral faces as "medium approachable" (Capitão et al., 2011). These findings might also reflect the previous finding that individuals with WS have more difficulty than controls in interpreting facial expressions (Gagliardi et al., 2003; Plesa-Skwerer et al., 2006; Porter et al., 2007; Capitão et al., 2011), and have atypical eye scanpath patterns when viewing both positive and negative facial expressions (Porter et al., 2010).

The current findings are the first to suggest that individuals with WS can discriminate mild vs. extreme degrees of trustworthiness, albeit not as accurately as controls. The participants with WS made more errors on the mild than extreme faces for both the untrustworthy and trustworthy faces. The difficulty of discriminating degree of trustworthiness may be associated with the previous finding that individuals with WS make more errors than control participants when labeling facial expressions (Gagliardi et al., 2003; Plesa-Skwerer et al., 2006; Porter et al., 2007; Jarvinen-Pasley et al., 2010; Capitão et al., 2011).

Importantly, our findings also demonstrate that older individuals with WS, like the older control participants, are more likely to make correct approachability decisions than younger participants. There has been little examination of the development of hypersociability in WS. Martens et al. (2009) found that adults with WS were more likely to use typical facial features to determine approachability than children. Additional research is needed in order to investigate the development of hypersociability in WS more fully.

The data appear to contradict previous studies that have demonstrated that individuals with WS do not show an attention bias to angry faces (Dodd and Porter, 2010) and they rate faces depicting negative emotions, such as anger, disgust, and fear, as unapproachable (Frigerio et al., 2006). The reason for the conflicting evidence may depend in part on the nature of the task stimuli utilized in the various studies. The studies conducted by Bellugi et al. (1999), Fishman et al. (2011), Jones et al. (2000), and Martens et al. (2009) used facial stimuli that had been rated by the normative sample as untrustworthy and unapproachable (Adolphs et al., 1998). In contrast, the studies by Dodd and Porter (2010) and Frigerio et al. (2006) utilized facial stimuli that depicted angry and fearful faces. So while there is evidence that individuals with WS appear to be able to distinguish anger and fear, they may have more difficulty evaluating the approachability of faces that vary in degree of trustworthiness. This conclusion is further supported by evidence that individuals with WS have difficulty inferring complex emotions, including "don't trust," (Riby and Back, 2010).

We recognize that this mouse-tracking task requires fine motor coordination and visual control of spatially directed hand movements, skills which are difficult for many individuals with WS (Atkinson et al., 1997; Elliott et al., 2006; Hocking et al., 2011). Elliott and colleagues evaluated mouse cursor movements in four individuals with WS and found that they demonstrated slower RTs and had more errors than adults with other types of developmental disabilities. Slower RTs were also observed in adults with WS who used a stylus on a touchscreen to draw a horizontal line between circles that varied in size and in distance from one another (Hocking et al., 2011). In addition, individuals with WS have been shown to have difficulty posting a card through slots with various orientations (Atkinson et al., 1997). In the card post task, individuals with 
WS displayed awkward arm and hand postures as they attempted to rotate the card to match the slot's orientation. However, it seems unlikely that the spatial nature of our task was responsible for the pattern of responses that we obtained. We controlled for RT in our statistical analyses and the experiment was designed so that participants could click anywhere on the response circle (green to approach or red to avoid), so they did not have to click directly on a small target. Furthermore, the mouse-tracking task, although still requiring visuomotor control, did not require hand or arm rotations and the surface of the table might have helped stabilize their arm motor movements.

At this point we do not have data to measure the reliability of approachability ratings using a mouse-tracking paradigm compared to traditional Likert-scale ratings, but this is an area we plan to investigate in future studies. In addition, our MD findings are limited in that the mouse-tracking software does not afford automated time-course analyses in order to more closely examine the pattern of the trajectory. Now that mouse-tracking software has been utilized in WS using automated metrics, it would be interesting for future studies to expand the current findings by conducting time series analyses of the trajectories. It would also be important to evaluate the ecological validity of the mouse-tracking task using complementary in vivo studies of sociability. Such studies might include showing faces that vary in degrees of trustworthiness on two video screens, and asking individuals with WS to walk toward the video screen of the person that they wish to approach. Showing physical movement toward a target would be a logical extension of the trajectory of a computer mouse.

Although the outgoing and friendly nature of individuals with WS makes them endearing, parents are well aware that this aspect of their personality makes them extremely vulnerable to exploitation. We are the first to use mouse-tracking methodology to examine hypersociability in WS and believe this that methodology increases our understanding of the continuous cognitive processes that may underlie hypersociability in WS. Mouse-tracking trajectory data have been validated using simulated trajectories and other RT data (Freeman and Ambady, 2010). Rather than relying on Likert-style tasks which rely only on end point decisions, mouse-tracking data allow us to view the dynamics of approachability decisions and to compare these dynamics between individuals with WS and controls. Mouse-tracking trajectories which depict a fairly straight trajectory between the starting point (i.e., viewing an untrustworthy face) and the final choice (choosing to avoid) suggest that the decision is fairly firm and the person is not strongly considering approaching the face. On the other hand,

\section{REFERENCES}

Adolphs, R. (1995). Fear and the human amygdala. Nature 372, 669-672.

Adolphs, R. (2003). Is the human amygdala specialized for processing social information? Ann. N. Y. Acad. Sci. 985, 326-340.

Adolphs, R., Tranel, D., and Damasio, A. (1998). The human amygdala in social judgment. Nature 393, 470-474.

Atkinson, J., King, J., Braddick, O., Nokes, L., Anker, S., and

mouse-tracking trajectories which deviate a great deal toward approaching an untrustworthy face before finally deciding to avoid the face inform us about the dynamic nature of some approachability decisions and help characterize the hypersociability noted in WS.

These findings may also assist in the development of interventions that can improve the emotion processing skills of individuals with WS. For example, individuals with WS could be taught to discriminate how facial features change from trustworthy to untrustworthy (i.e., the inner portion of the eyebrows lower and the corners of the mouth turn down). Mouse-tracking could then be used to determine if this type of intervention training influences mouse trajectories as decisions of approachability are made. Research suggests that interventions can be successful in helping individuals improve their recognition of facial expressions (Dadds et al., 2006). Individuals who are taught to accurately detect and identify facial expressions demonstrate increased positive social interactions and decrease self-reported feelings of anxiety (Izard et al., 2001). There is increasing evidence that deficits in emotion perception can be remediated among individuals with developmental disabilities. Remediation in correctly perceiving emotions from facial cues has resulted in improved emotion perception in adults with intellectual disabilities. Importantly, these findings have generalized from viewing photographs to viewing videotaped role plays and have persisted for at least nine months (McAlpine et al., 1992). Similarly, training individuals with autism to correctly process facial expressions resulted in brain activation changes in regions underlying facial processing (Bolte et al., 2006). Behavioral outcome measures have been used to verify the benefits of emotion processing training (Radice-Neumann et al., 2009) and therefore mouse-tracking paradigms may be beneficial in evaluating the effectiveness of emotion processing interventions and their effects on hypersociability in individuals with WS.

\section{ACKNOWLEDGMENTS}

We would like to thank the Williams syndrome and control participants for their willingness to participate in this study. We would also like to acknowledge the Williams Syndrome Association for their assistance in coordinating the research. Thank you also to two anonymous reviewers, and to Brad Okdie and Melissa Buelow, for their helpful comments on this manuscript. This work was supported by The Ohio State University-Newark Impacts for Excellence Grant (Dr. Martens) and by The Ohio State University Nisonger Center (Dr. Martens, Dr. Andridge).

we animate the fusiform gyrus? Behav. Neurosci. 120, 211-216.

Brock, J. (2007). Language abilities in Williams syndrome: a critical review. Dev. Psychopathol. 19, 97-127.

Capitão, L., Sampaio, A., Fernandez, M., Sousa, N., Pinheiro, A., and Goncalves, O. F. (2011). Williams syndrome hypersociability: a neuropsychological study of the amygdala and prefrontal cortex hypotheses. Res. Dev. Disabil. 32, 1169-1179.
Chapman, C. A., du Plessis, A., and Pober, B. R. (1996). Neurologic findings in children and adults with Williams syndrome. J. Child Neurol. 11, 63-65.

Clahsen, H., Ring, M., and Temple, C. (2004). "Lexical and morphological skills in English-speaking children with Williams syndrome," in Williams Syndrome Across Languages, eds S. Bartke and J. Siegmüller (Amsterdam: Benjamins), 221-244. 
Cowell, A. J., and Stanney, K. M. (2002). On manipulating nonverbal interaction style to increase anthropomorphic computer character credibility. J. Appl. Psychol. 61, 428-432.

Cunningham, J. G., and Odom, R. D. (1986). Differential salience of facial features in children's perception of affective expression. Child Dev. 57, 136-142.

Dadds, M. R., Perry, Y., Hawes, D. J., Merz, S., Riddell, A. C., Haines, D. J., Solak, E., and Abeygunawardane, A. I. (2006). Attention to the eyes and fear-recognition deficits in child psychopathy. Br. J. Psychiatry 189, 280-281.

Dale, R., Kehoe, C., and Spivey, M. J. (2007). Graded motor responses in the time course of categorizing atypical exemplars. Mem. Cognit. 35, 15-28.

Diggle, P. J., Heagerty, P., Liang, K. Y., and Zeger, S. L. (2002). Analysis of Longitudinal Data. Oxford: Clarendon Press.

Dodd, H. F., and Porter, M. A. (2010). I see happy people: attention bias towards happy but not angry facial expressions in Williams syndrome. Cogn. Neuropsychiatry 15, 549-567.

Doherty-Sneddon, G., Riby, D. M., Calderwood, L., and Ainsworth, L. (2009). Stuck on you: faceto-face arousal and gaze aversion in Williams syndrome. Cogn. Neuropsychiatry 14, 510-523.

Don, A. J., Schellenberg, E. G., and Rourke, B. P. (1999). Music and language skills of children with Williams syndrome. Child Neuropsychol. 5, 154-170.

Elliott, D., Welsh, T. N., Lyons, J., Hansen, S., and Wu, M. (2006). The visual regulation of goal-directed reaching movements in adults with Williams syndrome, Down syndrome, and other developmental delays. Motor Control 10, 34-54.

Fishman, I., Yam, A., Bellugi, U., and Mills, D. (2011). Language and sociability: insights from Williams syndrome. J. Neurodev. Disord. 3, 185-192.

Freeman, J. B., and Ambady, N. (2009). Motions of the hand expose the partial and parallel activation of stereotypes. Psychol. Sci. 20, 1183-1188.

Freeman, J. B., and Ambady, N. (2010). MouseTracker: software for studying real-time mental processing using a computer mouse-traacking method. Behav. Res. Methods 42, 226-241.

Freeman, J. B., Ambady, N., Rule, N. O., and Johnson, K. L. (2008). Will a category cue attract you? Motor output reveals dynamic competition across person construal. J. Exp. Psychol. Gen. 137, 673-690.

Freeman, J. B., Pauker, K., Apfelbaum, E. P., and Ambady, N. (2010). Continuous dynamics in the real-time perception of race. J. Exp. Soc. Psychol. 46, 179-185.

Frigerio, E., Burt, D. M., Gagliardi, C., Cioffi, G., Martelli, S., Perrett, D. I., and Borgatti, R. (2006). Is everybody always my friend? Perception of approachability in Williams syndrome. Neuropsychologia 44, 254-259.

Gagliardi, C., Frigerio, E., Burt, D. M., Cazzaniga, I., Perrett, D. I., and Borgatti, R. (2003). Facial expression recognition in Williams syndrome. Neuropsychologia 41, 733-738.

Gold, J. I., and Shadlen, M. N. (2001). Neural computations that underlie decisions about sensory stimuli. Trends Cogn. Sci. (Regul. Ed.) 5, $10-16$.

Gosch, A., Stading, G., and Pankau, R. (1994). Linguistic abilities in children with Williams-Beuren syndrome. Am. J. Med. Genet. 52, 291-296.

Haas, B. W., Mills, D., Yam, A., Hoeft, F., Bellugi, U., and Reiss, A. (2009). Genetic influences on sociability: heightened amygdala reactivity and event-related responses to positive social stimuli in Williams syndrome. J. Neurosci. 29, 1132-1139.

Hocking, D. R., Rinehart, N. J., McGinley, J. L., Moss, S. A., and Bradshaw, J. L. (2011). A kinematic analysis of visually-guided movement in Williams syndrome. J. Neurol. Sci. 301, 51-58.

Inversions, S. (2007). FaceGen Main Software Development Kit, Version 3.2 Edn. Vancouver, BC: Singular Inversions.

Izard, C., Fine, S., Schultz, D., Mostow, A., Ackerman, B., and Youngstrom, E. (2001). Emotion knowledge as a predictor of social behavior and academic competence in children at risk. Psychol. Sci. 12, 18-23.

Jarvinen-Pasley, A., Adolphs, R., Yam, A., Hill, K. J., Grichanik, M., Reilly, J., Mills, D., Reiss, A. L., Korenberg, J. R., and Bellugi, U. (2010). Affiliative behavior in Williams syndrome: social perception and reallife social behavior. Neuropsychologia 48, 2110-2119.

Jones, W., Bellugi, U., Lai, Z., Chiles, M., Reilly, J., Lincoln, A., and Adolphs, R. (2000). Hypersociability in Williams Syndrome. J. Cogn. Neurosci. 12, 30-46.
Karmiloff-Smith, A., Klima, E., Bellugi, U., Grant, J., and Baron-Cohen, S. (1995). Is there a social module? Language, face processing, and theory of mind in individuals with Williams Syndrome. J. Cogn. Neurosci. 7, 196-209.

Kaufman, A. S., and Kaufman, N. L. (2004). Kaufman Brief Intelligence Test, 2nd Edn. Circle Pines: American Guidance Services.

Kenward, M. G., and Roger, J. H. (1997). Small sample inference for fixed effects from restricted maximum likelihood. Biometrics 53 , 983-997.

Levy, Y., Pluber, H., and Bentin, S. (2011). Covert processing of facial expressions by people with Williams syndrome. Cortex 47, 23-34.

Lukács, Á., Racsmány, M., and Pléh, C. (2001). Vocabulary and morphological patterns in Hungarian children with Williams syndrome: a preliminary report. Acta Linguist. Hung. 48 243-269.

Magnuson, J. S. (2005). Moving hand reveals dynamics of thought. Proc. Natl. Acad. Sci. U.S.A. 102, 9995-9996.

Martens, M. A., Wilson, S. J., Dudgeon, P., and Reutens, D. C. (2009). Approachability and the amygdala: insights from Williams syndrome. Neuropsychologia 47, 2446-2453.

McAlpine, C., Singh, N. N., Ellis, C. R., Kendall, K. A., and Hampton, C. (1992). Enhancing the ability of adults with mental retardation to recognize facial expressions of emotion. Behav. Modif. 16, 559-573.

Mervis, C. B., and Klein-Tasman, B. P. (2000). Williams syndrome: cognition, personality, and adaptive behavior. Ment. Retard. Dev. Disabil. Res. Rev. 6, 148-158.

Mervis, C. B., Morris, C. A., KleinTasman, B. P., Bertrand, J., Kwitny, S., Appelbaum, L. G., and Rice, C. E. (2003). Attentional characteristics of infants and toddlers with Williams syndrome during triadic interactions. Dev. Neuropsychol. 23, 243-268.

Meyer-Lindenberg, A., Hariri, A. R. Munoz, K. E., Mervis, C. B., Mattay, V. S., Morris, C. A., and Berman, K. F. (2005). Neural correlates of genetically abnormal social cognition in Williams syndrome. Nat. Neurosci.8, 991-993.

Oosterhof, N. N., and Todorov, A. (2008). The functional basis of face evaluation. Proc. Natl. Acad. Sci. U.S.A. 105, 11087-11092.
Peoples, R., Franke, Y., Wang, Y., PerezJurado, L., Paperna, T., Cisco, M., and Francke, U. (2000). A physical map, including a BAC/PAC clone contig, of the Williams-Beuren syndromedeletion region at $7 \mathrm{q} 11.23$. Am. J. Hum. Genet. 66, 47-68.

Phelps, E. A. (2006). Emotion and cognition: insights from studies of the human amygdala. Annu. Rev. Psychol. 57, 27-53.

Plesa-Skwerer, D., Borum, L., Verbalis, A., Schofield, C., Crawford, N., Ciciolla, L., and Tager-Flusberg, H. (2009). Automatic responses to dynamic displays of facial expressions in adolescents and adults with Williams syndrome. Soc. Cogn. Affect. Neurosci. 4, 93-100.

Plesa-Skwerer, D., Faja, S., Schofield, C. Verbalis, A., and Tager-Flusberg, $\mathrm{H}$. (2006). Perceiving facial and vocal expressions of emotion in individuals with Williams syndrome. Am. J. Ment. Retard. 111, 15-26.

Porter, M. A., and Coltheart, M. (2006). Global and local processing in Williams syndrome, autism, and Down syndrome: perception, attention, and construction. Dev. Neu ropsychol. 30, 771-789.

Porter, M. A., Coltheart, M., and Langdon, R. (2007). The neuropsychological basis of hypersociability in Williams and Down syndrome. Neuropsychologia 45, 2839 -2849.

Porter, M. A., Shaw, T. A., and Marsh, P. J. (2010). Anusual attraction to the eyes in Williams-Beuren syndrome: a manipulation of facial affect while measuring face scanpaths. Cogn. Neuropsychiatry 15, 505-530.

Radice-Neumann, D., Zupan, B., Tomita, M., and Willer, B. (2009). Training emotional processing in persons with brain injury. J. Head Trauma Rehabil. 24, 313-323.

Riby, D. M., and Back, E. (2010). Can individuals with Williams syndrome interpret mental states from moving faces? Neuropsychologia 48, 1914-1922.

Riby, D. M., Jones, N., Brown, P. H., Robinson, L. J., Langton, S. R. H., Bruce, V., and Riby, L. M. (2011). Attention to faces in Williams syndrome. J. Autism Dev. Disord. 41, 1228-1239.

Robinson, B. F., Mervis, C. B., and Robinson, B. W. (2003). The roles of verbal short-term memory and working memory in the acquisition of grammar by children with Williams syndrome. Dev. Neuropsychol. 23, 13-31. 
Santos, A., Silva, C., Rosset, D., and Deruelle, C. (2010). Just another face in the crowd: evidence for decreased detection of angry faces in children with Williams syndrome. Neuropsychologia 48, 1071-1078.

Shin, J. C., and Rosenbaum, D. A. (2002). Reaching while calculating: scheduling of cognitive and perceptual-motor processes. J. Exp. Psychol. Gen. 131, 206-219.

Spivey, M. J., and Dale, R. (2006). Continuous dynamics in real-time cognition. Curr. Dir. Psychol. Sci. 15, 207-211.

Spivey, M. J., Grosjean, M., and Knoblich, G. (2005). Continuous attraction toward phonological competitors. Proc. Natl. Acad. Sci. U.S.A. 102, 10393-10398.

Stromme, P., Bjornstad, P. G., and Ramstad, K. (2002). Prevalence estimation of Williams syndrome. J. Child Neurol.17, 269-271.
Todorov, A., Said, C. P., Engell, A. D., and Oosterhof, N. N (2008). Understanding evaluation of faces on social dimensions. Trends Cogn. Sci. (Regul. Ed.) 12, 455-460.

Vicari, S., Bates, E., Caselli, M. C., Pasqualetti, P., Gagliardi, C., Tonucci, R., and Volterra, V. (2004). Neuropsychological profile of Italians with Williams syndrome: an example of a dissociation between language and cognition? J. Int. Neuropsychol. Soc. 10, 862-876.

Volterra, V., Capirci, O., Pezzini, G., Sabbadini, L., and Vicari, S. (1996). Linguistic abilities in Italian children with Williams syndrome. Cortex 32, 663-677.

Volterra, V., Caselli, M. C., Capirci, O., Tonucci, F., and Vicari, S. (2003). Early linguistic abilities of Italian children with Williams syndrome. Dev. Neuropsychol. 23, 33-58.
Want, S. C., Pascalis, O., Coleman, M., and Blades, M. (2003). Recognizing people from the inner or outer parts of their faces: developmental data concerning “unfamiliar" faces. $\mathrm{Br}$. J. Dev. Psychol. 21, 125-135.

Willis, J., and Todorov, A. (2006). First impressions: making up your mind after $100 \mathrm{~ms}$ exposure to a face. Psychol. Sci. 17, 592-598.

Winston, J. S., Strange, B. A., O'doherty, J., and Dolan, R. J. (2002). Automatic and intentional brain responses during evaluation of trustworthiness of faces. Nat. Neurosci. 5, 277-283.

Wojnowicz, M. T., Ferguson, M. J., Dale, R., and Spivey, M. J. (2009). The selforganization of explicit attitudes. Psychol. Sci. 20, 1428-1435.

Conflict of Interest Statement: The authors declare that the research was conducted in the absence of any commercial or financial relationships that could be construed as a potential conflict of interest.

Received: 25 January 2012; accepted: 04 May 2012; published online: 04 June 2012.

Citation: Martens MA, Hasinski AE Andridge $R R$ and Cunningham WA (2012) Continuous cognitive dynamics of the evaluation of trustworthiness in Williams syndrome. Front. Psychology 3:160. doi: 10.3389/fpsyg.2012.00160 This article was submitted to Frontiers in Developmental Psychology, a specialty of Frontiers in Psychology.

Copyright (C) 2012 Martens, Hasinski, Andridge and Cunningham. This is an open-access article distributed under the terms of the Creative Commons Attribution Non Commercial License, which permits non-commercial use, distribution, and reproduction in other forums, provided the original authors and source are credited. 